\title{
APPROXIMATION METHOD AND EQUILIBRIA OF ABSTRACT ECONOMIES
}

\author{
KOK-KEONG TAN AND XIAN-ZHI YUAN
}

(Communicated by Dale Alspach)

\begin{abstract}
By applying an equilibrium existence theorem for a qualitative game due to Ding and Tan and by employing an "approximation" method used by Tulcea, we obtain an equilibrium existence theorem for an abstract economy (generalized game) in which the constraint correspondences are not assumed to have open graphs nor open lower sections (which are generally assumed in the literature). Our result generalizes the corresponding results of Shafer and Sonnenschein (1975), Borglin and Keiding (1976), Yannelis and Prabhakar (1983), Tulcea (1986), and Chang (1990) in several ways.
\end{abstract}

\section{INTRODUCTION}

Let $E$ be a vector space and $A \subset E$. We shall denote by $\operatorname{co} A$ the convex hull of $A$. If $A$ is a subset of a topological space $X$, the closure of $A$ in $X$ is denoted by $\operatorname{cl}_{X} A$ and the interior of $A$ in $X$ is denoted by $\operatorname{int}_{X}(A)$. Also, $A$ is said to be compactly open in $X$ if, for each nonempty compact subset $C$ of $X, A \cap C$ is open in $C$. Let $X$ be a set; we shall denote by $2^{X}$ the family of all subsets of $X$. Let $X$ and $Y$ be sets and $F: X \rightarrow 2^{Y}$. Then the graph of $F$, denoted by $\operatorname{Graph}(F)$, is the set $\{(x, y) \in X \times Y: y \in F(x)\}$. Suppose $X$ and $Y$ are topological spaces and $F: X \rightarrow 2^{Y}$. Then

(1) $F$ is said to have an open graph if $\operatorname{Graph}(F)$ is open in $X \times Y$;

(2) the map $\bar{F}: X \rightarrow 2^{Y}$ is defined by

$$
\bar{F}(x)=\left\{y \in Y:(x, y) \in \operatorname{cl}_{X \times Y} \operatorname{Graph}(F)\right\}
$$

for each $x \in X$ (the set $\operatorname{cl}_{X \times Y} \operatorname{Graph}(F)$ is called the adherence of the graph of $T)$;

(3) $F$ is said to be lower semicontinuous (respectively, upper semicontinuous) if for each closed (respectively, open) subset $C$ of $Y$ the set $\{x \in X$ : $F(x) \subset C\}$ is closed (respectively, open) in $X$; and

Received by the editors January 12, 1993.

1991 Mathematics Subject Classification. Primary 47H04, 47N10, 49J45, 90A14, 90D06, 90D13.

Key words and phrases. Compactly open, open graph, closed graph, lower semicontinuous, upper semicontinuous, class $L_{C}, L_{C}$-majorant, $L_{C}$-majorized, one-person game, qualitative game, generalized game, abstract economy, equilibrium point, paracompact, topological vector space, locally convex space. 
(4) $F$ is said to have open lower sections if $F^{-1}(y):=\{x \in X: y \in F(x)\}$ is open in $X$ for each $y \in Y$.

Let $X$ be a topological space, $Y$ a nonempty subset of a vector space $E$, $\theta: X \rightarrow E$ a map, and $\phi: X \rightarrow 2^{Y}$ a correspondence. Then

(1) $\phi$ is said to be of class $L_{\theta, C}$ if, for every $x \in X, \operatorname{co} \phi(x) \subset Y$ and $\theta(x) \notin \operatorname{co} \phi(x)$ and, for each $y \in Y, \phi^{-1}(y)=\{x \in X: y \in \phi(x)\}$ is compactly open in $X$; and

(2) a correspondence $\phi_{x}: X \rightarrow 2^{Y}$ is said to be an $L_{\theta, C}$-majorant of $\phi$ at $x \in X$ if there exists an open neighborhood $N_{x}$ of $x$ in $X$ such that

(a) for each $z \in N_{x}, \phi(z) \subset \phi_{x}(z)$ and $\theta(z) \notin \operatorname{co} \phi_{x}(z)$,

(b) for each $z \in X, \operatorname{co} \phi_{x}(z) \subset Y$, and

(c) for each $y \in Y, \phi_{x}^{-1}(y)$ is compactly open in $X$;

(3) $\phi$ is $L_{\theta, C}$-majorized if for each $x \in X$ with $\phi(x) \neq \varnothing$ there exists an $L_{\theta, C}$-majorant of $\phi$ at $x$ in $X$.

Our notions of correspondences being of class $L_{\theta, C}, L_{\theta, C}$-majorant of $\phi$ at $x$, and $L_{\theta, C}$-majorized generalize the corresponding notions of correspondences being of class $L, \mathscr{L}$-majorant of $\phi$ at $x$, and $\mathscr{L}$-majorized introduced by Yannelis and Prabhakar [10, p. 240]. As in [4], we shall only deal with either the case (I) $X=Y$ and is a nonempty convex subset of a topological vector space and $\theta=I_{X}$, the identity map on $X$, or the case (II) $X=\prod_{i \in I} X_{i}$ and $\theta=\pi_{j}: X \rightarrow X_{j}$ is the projection of $X$ onto $X_{j}$ and $X_{j}=Y$ is a nonempty convex subset of a topological vector space. In both cases (I) and (II), we shall write $L_{C}$ in place of $L_{\theta, C}$.

A one-person game is a quadruple $(X ; A, B ; P)$ where $X$ is a topological space, $A, B: X \rightarrow 2^{X}$ are constraint correspondences, and $P: X \rightarrow 2^{X}$ is a preference correspondence. An equilibrium point for $(X ; A, B ; P)$ is a point $x^{*} \in X$ such that $x^{*} \in \bar{B}\left(x^{*}\right)$ and $A\left(x^{*}\right) \cap P\left(x^{*}\right)=\varnothing$.

Let $I$ be a (finite or infinite) set of agents (players). An abstract economy (generalized game) is a family $\Gamma=\left(X_{i} ; A_{i}, B_{i} ; P_{i}\right)_{i \in I}$ of quadruples $\left(X_{i} ; A_{i}, A_{i} ; P_{i}\right)$ where, for each $i \in I, X_{i}$ is a topological space, $A_{i}, B_{i}$ : $X:=\prod_{j \in I} X_{j} \rightarrow 2^{X_{i}}$ are constraint correspondences, and $P_{i}: X \rightarrow 2^{X_{i}}$ is a preference correspondence. An equilibrium point for $\Gamma$ is a point $x^{*} \in X$ such that, for each $i \in I, x_{i}^{*}=\pi_{i}\left(x^{*}\right) \in \overline{B_{i}}\left(x^{*}\right)$ and $A_{i}\left(x^{*}\right) \cap P_{i}\left(x^{*}\right)=\varnothing$ where $\pi_{i}: X \rightarrow X_{i}$ is the projection. We remark that when $\overline{B_{i}}\left(x^{*}\right)=\operatorname{cl}_{X_{i}} B_{i}\left(x^{*}\right)$ (which is the case when $B_{i}$ has a closed graph in $X \times X_{i}$, in particular, when $\mathrm{cl}_{X_{i}} B_{i}$ is upper semicontinuous with closed values) for each $i \in I$ our definition of an equilibrium point for an abstract economy (generalized game) coincides with that of [4]. Also, according to Gale and Mas-Colell [5], a qualitative game is a family $\Gamma=\left(X_{i}, P_{i}\right)_{i \in I}$ of ordered pairs $\left(X_{i}, P_{i}\right)$ where, for each $i \in I, X_{i}$ is a topological space and $P_{i}: X=\prod_{j \in I} X_{j} \rightarrow 2^{X_{i}}$ is an irreflexive preference correspondence, i.e., $x_{i} \notin P_{i}(x)$ for all $x \in X$. A point $x^{*} \in X$ is said to be an equilibrium point of the qualitative game $\Gamma$ if $P_{i}\left(x^{*}\right)=\varnothing$ for all $i \in I$.

In this paper we first prove one useful lemma. Next by applying an equilibrium existence theorem for a qualitative game due to Ding and Tan [4] and by employing an "approximation" method used by Tulcea [8], we obtain an equilibrium existence theorem for an abstract economy (generalized game). Our results generalize the corresponding results of Chang [3], Yannelis and Prab- 
haker [10], Borglin and Keiding [2], Shafer and Sonnenschein [7], and Tulcea [8] in several ways.

\section{Preliminaries}

We shall need the following simple result.

Lemma 2.1. Let $X$ be a topological space, $Y$ a nonempty subset of a topological vector space $E, \mathscr{B}$ a base for the zero neighborhoods in $E$, and $B: X \rightarrow 2^{Y}$. For each $V \in \mathscr{B}$, let $B_{V}: X \rightarrow 2^{Y}$ be defined by $B_{V}(x)=(B(x)+V) \cap Y$ for each $x \in X$. If $\hat{x} \in X$ and $\hat{y} \in Y$ are such that $\hat{y} \in \bigcap_{V \in \mathscr{B}} \overline{B_{V}}(\hat{x})$, then $\hat{y} \in \bar{B}(\hat{x})$.

Proof. Suppose $\hat{y} \notin \bar{B}(\hat{x})$. Then $(\hat{x}, \hat{y}) \notin \operatorname{cl}_{X \times Y} \operatorname{Graph}(B)$. Let $U$ be an open neighborhood of $\hat{x}$ in $X$ and $V \in \mathscr{B}$ be such that

$$
(U \times(\hat{y}+V)) \cap \operatorname{Graph}(B)=\varnothing .
$$

Choose $W \in \mathscr{B}$ such that $W-W \subset V$. Since $\hat{y} \in \overline{B_{W}}(\hat{x})$, by assumption, $(\hat{x}, \hat{y}) \in \operatorname{cl}_{X \times Y} \operatorname{Graph}\left(B_{W}\right)$ so that $(U \times(\hat{y}+W)) \cap \operatorname{Graph}\left(B_{W}\right) \neq \varnothing$. Take any $x \in U$ and $w_{1} \in W$ with $\left(x, \hat{y}+w_{1}\right) \in \operatorname{Graph}\left(B_{W}\right)$ so that $\hat{y}+w_{1} \in B_{W}(x)=$ $(B(x)+W) \cap Y$. Let $z \in B(x)$ and $w_{2} \in W$ be such that $y+w_{1}=z+w_{2} \in Y$. It follows that $z=\hat{y}+w_{1}-w_{2} \in \hat{y}+W-W \subset \hat{y}+V$, so $(\hat{y}+V) \cap B(x) \neq \varnothing$ where $x \in U$. This contradicts $(*)$. Thus we must have $\hat{y} \in \overline{B_{i}}(\hat{x})$.

We shall also need Theorem 1 and Theorem 3 of [4], which are stated below as Lemma 2.2 and Lemma 2.3 respectively.

Lemma 2.2. Let $X$ be a nonempty paracompact convex subset of a topological vector space and $P: X \rightarrow 2^{X} L_{C}$-majorized. Suppose that there exist $a$ nonempty compact convex subset $X_{0}$ of $X$ and a nonempty compact subset $K$ of $X$ such that for each $y \in X \backslash K$ there is an $x \in \operatorname{co}\left(X_{0} \cup\{y\}\right)$ with $x \in \operatorname{co} P(y)$. Then there exists an $\hat{x} \in K$ such that $P(\hat{x})=\varnothing$.

Lemma 2.2 generalizes Theorem 5.1 and also Corollary 5.1 of Yannelis and Prabhakar [10] in the following ways:

(i) The space $X$ is paracompact instead of being compact.

(ii) The correspondence $P$ is $\mathscr{L}_{C}$-majorized instead of being class $\mathscr{L}$ or $\mathscr{L}$-majorized.

Lemma 2.3. Let $\Gamma=\left(X_{i}, P_{i}\right)_{i \in I}$ be a qualitative game such that $X=\prod_{i \in I} X_{i}$ is paracompact. Suppose the following conditions are satisfied:

(i) For each $i \in I, X_{i}$ is a nonempty convex subset of a topological vector space.

(ii) For each $i \in I, P_{i}: X \rightarrow 2^{X_{i}}$ is $L_{C}$-majorized.

(iii) $\bigcup_{i \in I}\left\{x \in X: P_{i}(x) \neq \varnothing\right\}=\bigcup_{i \in I}$ int $_{X}\left\{x \in X: P_{i}(x) \neq \varnothing\right\}$.

(iv) There exist a nonempty compact convex subset $X_{0}$ of $X$ and a nonempty compact subset $K$ of $X$ such that for each $y \in X \backslash K$ there is an $x \in \operatorname{co}\left(X_{0} \cup\{y\}\right)$ with $x_{i} \in \operatorname{co} P_{i}(y)$ for all $i \in I$.

Then $\Gamma$ has an equilibrium point in $K$.

\section{EQUILIBRIUM POINTS}

In this section we shall use the approximation technique used by Tulcea [8] to obtain equilibrium existence theorems for abstract economies. First we shall 
prove the following existence theorem of "approximate" equilibrium points for a one-person game.

Theorem 3.1. Let $X$ be a nonempty paracompact convex subset of a topological vector space $E$ and $A, B, P: X \rightarrow 2^{X}$ be such that

(i) $A$ is lower semicontinuous and, for each $x \in X, A(x)$ is nonempty and $\operatorname{co} A(x) \subset B(x)$;

(ii) $A \cap P$ is $L_{C}$-majorized;

(iii) there exist a nonempty compact convex subset $X_{0}$ of $X$ and a nonempty compact subset $K$ of $X$ such that, for each $y \in X \backslash K$,

$$
\operatorname{co}\left(X_{0} \cup\{y\}\right) \cap \operatorname{co}(A(y) \cap P(y)) \neq \varnothing .
$$

Then for each open convex neighborhood $V$ of zero in $E$ the one-person game $\left(X ; A, \overline{B_{V}} ; P\right)$ has an equilibrium point in $K$; i.e., there exists a point $x_{V} \in K$ such that $x_{V} \in \overline{B_{V}}\left(x_{V}\right)$ and $A\left(x_{V}\right) \cap P\left(x_{V}\right)=\varnothing$ where $B_{V}(x)=(B(x)+V) \cap X$ for each $x \in X$.

Proof. Let $V$ be an open convex neighborhood of zero in $E$, and define $A_{V}, B_{V}: X \rightarrow 2^{X}$ by $A_{V}(x)=(A(x)+V) \cap X$ and $B_{V}(x)=(B(x)+V) \cap X$ for each $x \in X$. Since $A$ is lower semicontinuous, $A_{V}$ has an open graph in $X \times X$ by Lemma 4.1 of $\left[3\right.$, p. 244] or [8, p. 7]. By (i), $A_{V}(x) \subset B_{V}(x)$ for each $x \in X$. Let $F_{V}=\left\{x \in X: x \notin \overline{B_{V}}(x)\right\}$. Then $F_{V}$ is open in $X$. Define $\Psi_{V}: X \rightarrow 2^{X}$ by

$$
\Psi_{V}= \begin{cases}A(x) \cap P(x) & \text { if } x \notin F_{V}, \\ A_{V}(x) & \text { if } x \in F_{V} .\end{cases}
$$

Suppose $x \in X$ is such that $\Psi_{V}(x) \neq \varnothing$.

Case 1: Suppose $x \in F_{V}$. Let $\Phi_{x}=A_{V}$ and $N_{x}=F_{V}$. Then $N_{x}$ is an open neighborhood of $x$ in $X$ such that

(a) for each $z \in N_{x}, \Psi_{V}(z)=A_{V}(z)=\Phi_{x}(z), z \notin \overline{B_{V}}(z)$, so that $z \notin A_{V}(z)=\Phi_{x}(z)$

(b) for each $y \in X, \Phi_{x}^{-1}(y)=A_{V}^{-1}(y)$ is open in $X$ since $A_{V}$ has an open graph in $X \times X$.

Thus $\Phi_{x}$ is an $L_{C}$-majorant of $\Psi_{V}$ at $x$.

Case 2: Suppose $x \notin F_{V}$. Then $\Psi_{V}(x)=A(x) \cap P(x) \neq \varnothing$. Since $A \cap P$ is $L_{C}$-majorized by (ii), there exist an open neighborhood $N_{x}$ of $x$ in $X$ and a correspondence $\Phi_{x}^{\prime}: X \rightarrow 2^{X}$ such that

(a) $\Psi_{V}(z)=A(z) \cap P(z) \subset \Phi_{x}^{\prime}(z)$ and $z \notin \operatorname{co} \Phi_{x}^{\prime}(z)$ for each $z \in N_{x}$,

(b) for each $y \in X,\left(\Phi_{x}^{\prime}\right)^{-1}(y)$ is compactly open in $X$.

Define $\Phi_{x}: X \rightarrow 2^{X}$ by

$$
\Phi_{x}= \begin{cases}A_{V}(z) \cap \Phi_{x}^{\prime}(z) & \text { if } z \notin F_{V} \\ A_{V}(z) & \text { if } z \in F_{V}\end{cases}
$$

Note that

(i) for each $z \in N_{x}$, clearly $\Psi_{x}^{-1}(y) \subset \Phi_{x}(z)$ and it is easy to see that $z \notin \operatorname{co} \Phi_{x}(z)$,

(ii) for each $\left.y \in x, \Phi_{x}^{-1}(y)=\left[F_{V} \cup\left(\Phi_{x}^{\prime}\right)^{-1} y\right)\right] \cap A_{V}^{-1}(y)$ is compactly open in $X$.

Hence $\Phi_{x}$ is an $L_{C}$-majorant of $\Psi_{V}$ at $x$. 
Therefore, $\Psi_{V}$ is an $L_{C}$-majorized correspondence. Moreover by (iii), for each $y \in X \backslash K$, there exists $x \in \operatorname{co}\left(X_{0} \cup\{y\}\right) \cap \operatorname{co}(A(y) \cap P(y))$, so $x \in$ $\operatorname{co}(A(y) \cap P(y)) \subset \operatorname{co} \Psi_{V}(y)$. Thus by Lemma 2.2 there exists $\hat{x} \in K$ such that $\Psi_{V}(\hat{x})=\varnothing$. Since $A(\hat{x}) \neq \varnothing$ by (i), we must have $\hat{x} \in \overline{B_{V}}(\hat{x})$ and $A(\hat{x}) \cap P(\hat{x})=\varnothing$.

In the proof of Theorem 3.1, we obtained an equilibrium point of a oneperson game as a maximal element of a binary relation. This idea is originated from Aliprantis and Brown [1].

The following is an existence of an "approximate" equilibrium point for an abstract economy.

Theorem 3.2. Let $I$ be any (finite or infinite) set. For each $i \in I$, let $X_{i}$ be a nonempty convex subset of a topological vector space $E_{i}$ and $A_{i}, B_{i}, P_{i}: X=$ $\prod_{j \in I} X_{j} \rightarrow 2^{X_{i}}$ be such that

(a) $A_{i}$ is lower semicontinuous and, for each $x \in X, A_{i}(x)$ is nonempty and $\operatorname{co} A_{i}(x) \subset B_{i}(x)$

(b) $A_{i} \cap P_{i}$ is $L_{C}$-majorized;

(c) the set $E^{i}=\left\{x \in X:\left(A_{i} \cap P_{i}\right)(x) \neq \varnothing\right\}$ is open in $X$.

Suppose that $X$ is paracompact and that there exist a nonempty compact convex subset $X_{0}$ of $X$ and a nonempty compact subset $K$ of $X$ such that for each $y \in X \backslash K$ there is an $x \in \operatorname{co}\left(X_{0} \cup\{y\}\right)$ with $x_{i} \in \operatorname{co}\left(A_{i}(y) \cap P_{i}(y)\right)$ for all $i \in I$. Then given any $V=\prod_{i \in I} V_{i}$ where, for each $i \in I, V_{i}$ is an open convex neighborhood of zero in $E_{i}$, the abstract economy $\Gamma_{V}=\left(X_{i} ; A_{i}, B_{V_{i}} ; P_{i}\right)_{i \in I}$ has an equilibrium point in $K$; i.e., there exists a point $x_{V}=\left(x_{V_{i}}\right)_{i \in I} \in K$ such that $x_{V_{i}} \in \overline{B_{V_{i}}}\left(x_{V}\right)$ and $A_{i}\left(x_{V}\right) \cap P_{i}\left(x_{V}\right)=\varnothing$ for each $i \in I$ where $B_{V_{i}}=$ $\left(B_{i}(x)+V_{i}\right) \cap X_{i}$ for each $x \in X$ and each $i \in I$.

Proof. Let $V=\prod_{i \in I} V_{i}$ be given where, for each $i \in I, V_{i}$ is an open convex neighborhood of zero in $E_{i}$. Fix any $i \in I$, and define $A_{V_{i}}, B_{V_{i}}: X \rightarrow 2^{X_{i}}$ by $A_{V_{i}}(x)=\left(\operatorname{co} A_{i}(x)+V_{i}\right) \cap X_{i}$ and $B_{V_{i}}(x)=\left(B_{i}(x)+V_{i}\right) \cap X_{i}$ for each $x \in X$. By (a), $A_{i}$ is lower semicontinuous so that $\operatorname{co} A_{i}$ is also lower semicontinuous by Proposition 2.6 of [6, p. 366]. It follows from Lemma 4.1 of [3, p. 244] or from $\left[8\right.$, p. 7] that $A_{V_{i}}$ has an open graph in $X \times X$. Now let $F_{V_{i}}=\{x \in X$ : $\left.x_{i} \notin \overline{B_{V_{i}}}(x)\right\}$. Then $F_{V_{i}}$ is open in $X$. Define the map $Q_{V_{i}}: X \rightarrow 2^{X_{i}}$ by

$$
Q_{V_{i}}(x)= \begin{cases}\left(A_{i} \cap P_{i}\right)(x) & \text { if } x \notin F_{V_{i}}, \\ A_{i}(x) & \text { if } x \in F_{V_{i}} .\end{cases}
$$

We shall prove that the qualitative game $\mathscr{T}=\left(X_{i}, Q_{V_{i}}\right)_{i \in I}$ satisfies all conditions of Lemma 2.3. First we note that for each $i \in I$ the set

$$
\begin{aligned}
\left\{x \in X: Q_{V_{i}}(x) \neq \varnothing\right\} & =F_{V_{i}} \cup\left\{x \in X \backslash F_{V_{i}}: A_{i}(x) \cap P_{i}(x) \neq \varnothing\right\} \\
& =F_{V_{i}} \cup\left(\left(X \backslash F_{V_{i}}\right) \cap E^{i}\right)=F_{V_{i}} \cup E^{i}
\end{aligned}
$$

is open in $X$ by (c). Let $x \in X$ be such that $Q_{V_{i}}(x) \neq \varnothing$. We consider the following two cases.

Case 1: $x \in F_{V_{i}}$. Let $\Psi_{x}=A_{V_{i}}$ and $N_{x}=F_{V_{i}}$. Then $N_{x}$ is an open neighborhood of $x$ in $X$ such that

(i) $Q_{V_{i}}(z) \subset \Psi_{x}(z)$ and by (b) $z_{i} \notin \operatorname{co} \Psi_{x}(z)$ for each $z \in N_{x}$;

(ii) $\operatorname{co}_{x}(z) \subset X_{i}$ for each $z \in X$ by (b); 
(iii) $\Psi_{x}^{-1}(y)=A_{V_{i}}^{-1}(y)$ is open in $X$ for all $y \in X_{i}$ since $A_{V_{i}}$ has an open graph.

Therefore, $\Psi_{x}$ is an $L_{C}$-majorant of $Q_{V_{i}}$ at $x$.

Case 2: $x \notin F_{V_{i}}$. Since $Q_{V_{i}}(x)=\left(A_{i} \cap P_{i}\right)(x) \neq \varnothing$ and $A_{i} \cap P_{i}$ is $L_{C^{-}}$ majorized, there exist an open neighborhood $N_{x}$ of $x$ in $X$ and a correspondence $\phi_{x}: X \rightarrow 2^{X_{i}}$ such that

(i) $\left(A_{i} \cap P_{i}\right)(z) \subset \phi_{x}(z)$ and $z_{i} \notin \operatorname{co} \phi_{x}(z)$ for each $z \in N_{x}$;

(ii) $\operatorname{co} \phi_{x}(z) \subset X_{i}$ for each $z \in X$;

(iii) $\phi_{x}^{-1}(y)$ is compactly open in $X$ for each $y \in X_{i}$.

Define $\Psi_{x}: X \rightarrow 2^{X_{i}}$ by

$$
\Psi_{x}(z)= \begin{cases}A_{V_{i}}(z) \cap \phi_{x}(z) & \text { if } z \notin F_{V_{i}}, \\ A_{V_{i}}(z) & \text { if } z \in F_{V_{i}} .\end{cases}
$$

Note that as $\left(A_{i} \cap P_{i}\right)(z) \subset \phi_{x}(z)$ and $A_{i}(z) \subset A_{V_{i}}(z)$ for each $z \in N_{x}$, we have $Q_{V_{i}}(z) \subset \Psi_{x}(z)$ and $\operatorname{co} \Psi_{x}(z) \subset X_{i}$. It is easy to see that $z_{i} \notin \operatorname{co} \Psi_{x}(z)$ for all $z \in X$. Moreover, for any $y \in X_{i}$, the set

$$
\begin{aligned}
\Psi_{x}^{-1}(y) & =\left\{z \in X: y \in \Psi_{x}(z)\right\} \\
& =\left\{z \in X \backslash F_{V_{i}}: y \in \Psi_{x}(z)\right\} \cup\left\{z \in F_{V_{i}}: y \in \Psi_{x}(z)\right\} \\
& =\left\{z \in X \backslash F_{V_{i}}: y \in A_{V_{i}}(z) \cap \phi_{x}(z)\right\} \cup\left\{z \in F_{V_{i}}: y \in A_{V_{i}}(z)\right\} \\
& =\left[\left(X \backslash F_{V_{i}}\right) \cap A_{V_{i}}^{-1}(y) \cap \phi_{x}^{-1}(y)\right] \cup\left[F_{V_{i}} \cap A_{V_{i}}^{-1}(y)\right] \\
& =\left[\phi_{x}^{-1}(y) \cup F_{V_{i}}\right] \cap A_{V_{i}}^{-1}(y)
\end{aligned}
$$

is compactly open in $X$. Therefore, $\Psi_{x}$ is an $L_{C}$-majorant of $Q_{V_{i}}$ at point $x$.

Hence $Q_{V_{i}}$ is an $L_{C}$-majorized correspondence. Now by assumption there exist a nonempty compact convex subset $X_{0}$ of $X$ and a nonempty compact subset $K$ of $X$ such that for each $y \in X \backslash K$ there is an $x \in \operatorname{co}\left(X_{0} \cup\{y\}\right)$ with $x_{i} \in \operatorname{co}\left(A_{i}(y) \cap P_{i}(y)\right)$ for all $i \in I$. Note that if $y \notin F_{V_{i}}$, then $x_{i} \in$ $\operatorname{co}\left(A_{i}(y) \cap P_{i}(y)\right) \subset \operatorname{co} Q_{V_{i}}(y)$ and if $y \in F_{V_{i}}$, then $x_{i} \in \operatorname{co}\left(A_{i}(y) \cap P_{i}(y)\right) \subset$ $\operatorname{co}\left(A_{i}(x)\right)=\operatorname{co} Q_{V_{i}}(y)$. Thus, for each $i \in I, x_{i} \in \operatorname{co} Q_{V_{i}}(y)$. Moreover, the set $\left\{x \in X: Q_{V_{i}}(x) \neq \varnothing\right\}=F_{V_{i}} \cup\left\{x \in X \backslash F_{V_{i}}:\left(A_{i} \cap P_{i}\right)(x) \neq \varnothing\right\}=F_{V_{i}} \cup E^{i}$ is open in $X$ by (c). Therefore, all hypotheses of Lemma 2.3 are satisfied, so by Lemma 2.3 there exists a point $x_{V}=\left(x_{V_{i}}\right)_{i \in I} \in K$ such that $Q_{V_{i}}\left(x_{V}\right)=\varnothing$ for all $i \in I$. Since, for each $i \in I, A_{i}(x)$ is nonempty, we must have $x_{V_{i}} \in \overline{B_{V_{i}}}\left(x_{V}\right)$ and $A_{i}\left(x_{V}\right) \cap P_{i}\left(x_{V}\right)=\varnothing$.

By Theorem 3.2 and Lemma 2.1, we shall present the main result of this paper as

Theorem 3.3. Let $\mathscr{G}=\left(X_{i} ; A_{i}, B_{i} ; P_{i}\right)_{i \in I}$ be an abstract economy such that $X=\prod_{i \in I} X_{i}$ is paracompact. Suppose the following conditions are satisfied:

(a) For each $i \in I, X_{i}$ is a nonempty convex subset of a locally convex Hausdorff topological vector space $E_{i}$.

(b) For each $i \in I, A_{i}: X \rightarrow 2^{X_{i}}$ is lower semicontinuous such that, for each $x \in X, A_{i}(x)$ is nonempty and $\operatorname{co} A_{i}(x) \subset B_{i}(x)$.

(c) For each $i \in I, A_{i} \cap P_{i}$ is $L_{C}$-majorized.

(d) For each $i \in I$, the set $E^{i}=\left\{x \in X:\left(A_{i} \cap P_{i}\right)(x) \neq \varnothing\right\}$ is open in $X$. 
(e) There exist a nonempty compact convex subset $X_{0}$ of $X$ and a non-empty compact subset $K$ of $X$ such that for each $y \in X \backslash K$ there is an $x \in \operatorname{co}\left(X_{0} \cup\{y\}\right)$ with $x_{i} \in \operatorname{co}\left(A_{i}(y) \cap P_{i}(y)\right)$ for all $i \in I$.

Then $\mathscr{G}$ has an equilibrium point in $K$; i.e., there exists a point $\hat{x}=\left(\hat{x}_{i}\right)_{i \in I} \in$ $K$ such that, for each $i \in I, \hat{x}_{i} \in \overline{B_{i}}(\hat{x})$ and $A_{i}(\hat{x}) \cap P_{i}(\hat{x})=\varnothing$.

Proof. For each $i \in I$, let $\mathscr{B}_{i}$ be the collection of all open convex neighborhoods of zero in $E_{i}$ and $\mathscr{B}=\prod_{i \in I} \mathscr{B}_{i}$. Given any $V \in \mathscr{B}$, let $V=\prod_{j \in I} V_{j}$, where $V_{j} \in \mathscr{B}_{j}$ for each $j \in I$. By Theorem 3.2, there exists an $\hat{x}_{V} \in K$ such that $\hat{x}_{V_{i}} \in \overline{B_{V_{i}}}\left(\hat{x}_{V}\right)$ and $A_{i}\left(\hat{x}_{V}\right) \cap P_{i}\left(\hat{x}_{V}\right)=\varnothing$ for each $i \in I$, where $B_{V_{i}}(x)=\left(B_{i}(x)+V_{i}\right) \cap X_{i}$ for each $x \in X$. It follows that the set $Q_{V}:=\{x \in$ $K: x_{i} \in \overline{B_{V_{i}}}(x)$ and $\left.A_{i}(x) \cap P_{i}(x)=\varnothing\right\}$ is a nonempty closed subset of $K$ by (d).

Now we want to prove $\left\{Q_{V}\right\}_{V \in \mathscr{B}}$ has the finite intersection property. Let $\left\{V_{1}, \ldots, V_{n}\right\}$ be any finite subset of $\mathscr{B}$. For each $i=1, \ldots, n$, let $V_{i}=$ $\prod_{j \in I} V_{i j}$ where $V_{i j} \in \mathscr{B}_{i}$ for each $j \in I$; let $V=\prod_{j \in I}\left(\bigcap_{i=1}^{n} V_{i j}\right)$. Then $Q_{V} \neq \varnothing$. Clearly $Q_{V} \subset \bigcap_{i=1}^{n} Q_{V_{i}}$ so that $\bigcap_{i=1}^{n} Q_{V_{i}} \neq \varnothing$. Therefore, the family $\left\{Q_{V}: V \in \mathscr{B}\right\}$ has the finite intersection property. Since $K$ is compact, $\bigcap_{V \in \mathscr{R}} Q_{V} \neq \varnothing$. Now take any $\hat{x} \in \bigcap_{V \in \mathscr{B}} Q_{V}$. Then, for each $i \in I, \hat{x}_{i} \in$ $\overline{B_{V_{i}}}(\hat{x})$ for each $V_{i} \in \mathscr{B}_{i}$ and $A_{i}(\hat{x}) \cap P_{i}(\hat{x})=\varnothing$. By Lemma 2.1, we also have, for each $i \in I, \hat{x}_{i} \in \overline{B_{i}}(\hat{x})$.

Corollary 3.4. Let $\mathscr{G}=\left(X_{i} ; A_{i}, B_{i} ; P_{i}\right)_{i \in I}$ be an abstract economy such that $X=\prod_{i \in I} X_{i}$ is paracompact. Suppose the following conditions are satisfied:

(a) For each $i \in I, X_{i}$ is a nonempty convex subset of a locally convex Hausdorff topological vector space $E_{i}$.

(b) For each $i \in I$ and for each $x \in X, A_{i}(x)$ is nonempty and $\operatorname{co} A_{i}(x) \subset$ $B_{i}(x)$.

(c) For each $i \in I, A_{i}$ and $P_{i}$ have open lower sections.

(d) For each $i \in I, A_{i} \cap P_{i}$ is $L_{C}$-majorized.

(e) There exist a nonempty compact convex subset $X_{0}$ of $X$ and a non-empty compact subset $K$ of $X$ such that for each $y \in X \backslash K$ there is an $x \in \operatorname{co}\left(X_{0} \cup\{y\}\right)$ with $x_{i} \in \operatorname{co}\left(A_{i}(y) \cap P_{i}(y)\right)$ for all $i \in I$.

Then $\mathscr{G}$ has an equilibrium point in $K$; i.e., there exists a point $\hat{x} \in K$ such that, for each $i \in I, \hat{x}_{i} \in \overline{B_{i}}(\hat{x})$ and $A_{i}(\hat{x}) \cap P_{i}(\hat{x})=\varnothing$.

Proof. By (c), the map $A_{i}: X \rightarrow 2^{X_{i}}$ is lower semicontinuous and the set $E^{i}=\left\{x \in X: A_{i}(x) \cap P_{i}(x) \neq \varnothing\right\}$ is open in $X$. Therefore, all the hypotheses in Theorem 3.3 are satisfied, so the conclusion follows.

Corollary 3.4 (and hence also Theorem 3.3) improves Theorem 6.1 of Yannelis and Prabhakar [10] in the following ways:

(i) the index set $I$ need not be countable;

(ii) for each $i \in I$, the set $X_{i}$ need not be metrizable;

(iii) for each $i \in I, A_{i} \cap P_{i}$ need not be $L$-majorized.

Corollary 3.5. Let $\mathscr{G}=\left(X_{i}, A_{i}, B_{i}, P_{i}\right)_{i \in I}$ be an abstract economy such that $X=\prod_{i \in I} X_{i}$ is paracompact. Suppose the following conditions are satisfied:

(a) For each $i \in I, X_{i}$ is a nonempty convex subset of a locally convex Hausdorff topological vector space. 
(b) For each $i \in I$ and for each $x \in X, A_{i}(x)$ is nonempty and $\operatorname{co} A_{i}(x) \subset$ $B_{i}(x)$.

(c) For each $i \in I, A_{i}$ has an open graph in $X \times X_{i}$ (respectively, is lower semicontinuous) and $P_{i}$ is lower semicontinuous (respectively, has an open graph in $X \times X_{i}$ ).

(d) For each $i \in I, A_{i} \cap P_{i}$ is $L_{C}$-majorized.

(e) There exist a nonempty compact convex subset $X_{0}$ of $X$ and a nonempty compact subset $K$ of $X$ such that for each $y \in X \backslash K$ there is an $x \in \operatorname{co}\left(X_{0} \cup\{y\}\right)$ with $x_{i} \in \operatorname{co}\left(A_{i}(y) \cap P_{i}(y)\right)$ for all $i \in I$.

Then $\mathscr{G}$ has an equilibrium point in $K$, i.e., there exists a point $\hat{x} \in X$ such that, for each $i \in I, \hat{x}_{i} \in \overline{B_{i}}(\hat{x})$ and $A_{i}(\hat{x}) \cap P_{i}(\hat{x})=\varnothing$.

Proof. For each $i \in I$, since $A_{i}$ has an open graph in $X \times X_{i}$ (respectively, is lower semicontinuous) and $P_{i}$ is lower semicontinuous (respectively, has open graph in $\left.X \times X_{i}\right)$, the map $A_{i} \cap P_{i}: X \rightarrow 2^{X_{i}}$ is also lower semicontinuous by Lemma 4.2 of $\left[9\right.$, p. 103], so the set $E^{i}=\left\{x \in X: A_{i}(x) \cap P_{i}(x) \neq \varnothing\right\}$ is open in $X$. Therefore, all conditions of Theorem 3.3 are satisfied and the conclusion follows.

Corollary 3.5 (and hence also Theorem 3.3) generalizes Corollary 3 of Borglin and Keiding [2, p. 315], Theorem 4.1 of Chang [3, p. 247], Theorem of Shafer and Sonnenschein [7, p. 374], and Theorem 5 of Tulcea [8, p. 10].

\section{REFERENCES}

1. C. D. Aliprantis and D. J. Brown, Equilibria in markets with a Riesz space of commodities, J. Math. Econom. 11 (1983), 189-207.

2. A. Borglin and H. Keiding, Existence of equilibrium actions of equilibrium, "A note on the 'new' existence theorems", J. Math. Econom. 3 (1976), 313-316.

3. S. Y. Chang, On the Nash equilibrium, Soochow J. Math. 16 (1990), 241-248.

4. X. P. Ding and K. K. Tan, On equilibria of non-compact generalized games, J. Math. Anal. Appl. 177 (1993), 226-238.

5. D. Gale and A. Mas-Colell, On the role of complete, transitive preferences in equilibrium theory, Equilibrium and Disequilibrium in Economic Theory (G. Schwodiauer, ed.), Reidel, Dordrecht, 1978, pp. 7-14.

6. E. Michael, Continuous selections. I, Ann. of Math. (2) 63 (1956), 361-382.

7. W. Shafer and H. Sonnenschein, Equilibrium in abstract economies without ordered preferences, J. Math. Econom. 2 (1975), 345-348.

8. C. I. Tulcea, On the equilibriums of generalized games, The Center for Mathematical Studies in Economics and Management Science, paper No. 696, 1986.

9. N. C. Yannelis, Equilibria in noncoorperative models of competition, J. Econom. Theory 41 (1987), 96-111.

10. N. C. Yannelis and N. D. Prabhakar, Existence of maximal elements and equilibria in linear topological spaces, J. Math. Econom. 12 (1983), 233-245.

Department of Mathematics, Statistics and Computing Science, Dalhousie UniverSity, Halifax, Nova Scotia, CaNada B3H 3J5

E-mail address: kktanecs.dal.ca

Department of MAthematics, University of QueEnSLAND, BrLsbane 4072, Australia

E-mail address: xzyemaths.uq.oz.au 